\title{
Inference on the parameters and reliability characteristics of generalized inverted scale family of distributions based on records
}

\author{
Ajit Chaturvedi, Ananya Malhotra * \\ Department of Statistics, University of Delhi, India
}

\begin{abstract}
A generalized inverted scale family of distributions is considered. Two measures of reliability are discussed, namely $\rho(t)=P(X>t)$ and $P=P(X>Y)$. Point and interval estimation procedures are developed for the parameters, $\rho(t)$ and $P$ based on records. Two types of point estimators are developed - uniformly minimum variance unbiased estimators (UMVUES) and maximum likelihood estimators (MLES). A comparative study of different methods of estimation is done through simulation studies and asymptotic confidence intervals of the parameters based on MLE and log transformed MLE are constructed. Testing procedures are also developed for the parametric functions of the distribution and a real life example has been analysed for illustrative purposes.
\end{abstract}

Keywords Generalized inverted scale family of distributions, Point estimation, Interval estimation, Records, Simulation studies

AMS 2010 subject classifications 62N05, 62F10

DOI: $10.19139 /$ soic.v6i2.304

\section{Introduction}

A scale family of distributions plays an important role in reliability analysis with some of its most common members being exponential distribution, Rayleigh distribution, half-logistic distribution etc. Gupta and Kundu $(1999,2001 \mathrm{a}, 2001 \mathrm{~b})$ introduced the generalized exponential distribution. If $Y$ is an exponential random variable $(r v)$, then $X=\frac{1}{Y}$ has an inverted exponential distribution. Lin et al. (1989) and Dey (2007) discussed inverted exponential distribution (IED) to analyze lifetime data. Abouammoh and Alshingiti (2009) discussed generalized inverted exponential distribution (GIED) by introducing a shape parameter and discussed their statistical and reliability properties. Under Type II censoring, Krishna and Kumar (2012) estimated reliability characteristics of GIED. Potdar and Shirke $(2012,2013)$ discussed inference on the scale family of lifetime distributions based on progressively censored data and generalized inverted scale family of distributions respectively. In this paper, we develop UMVUES and MLES of the powers of the parameters and reliability functions of the generalized inverted scale family of distributions based on record data. The powers of the parameter are estimated as they appear in expressions for moments and the hazard rate of the distributions.

The reliability function $\rho(t)$ is defined as the probability that a system survives until time $t$. Thus, if the $r v X$ denotes the lifetime of an item or a system, then $\rho(t)=P(X>t)$. The reliability characteristic under stressstrength setup defined as $P=P(X>Y)$, is another measure of reliability function which represents the reliability of a system (or an item) of random strength (or supply) $X$ subject to random stress (or demand) $Y$. Thus, $P$ is a measure of system's performance. A lot of work has been done in the literature for the point estimation and testing of $\rho(t)$ and $P$. For example, Pugh (1963), Basu (1964), Bartholomew (1957, 1963), Tong (1974, 1975), Johnson (1975), Kelley et al. (1976), Sathe and Shah (1981), Chao (1982), Chaturvedi and Surinder (1999) developed

\footnotetext{
*Correspondence to: Ananya Malhotra (Email: malhotra.ananya3@gmail.com).
}

ISSN 2310-5070 (online) ISSN 2311-004X (print)

Copyright (C) 2018 International Academic Press 
inferential procedures for $\rho(t)$ and $P$ for exponential distribution. Constantine et al. (1986) derived UMVUE and MLE for $P$ associated with gamma distribution. Awad and Gharraf (1986) estimated $P$ for Burr distribution. For estimation of $\rho(t)$ corresponding to Maxwell and generalized Maxwell distributions, one may refer to Tyagi and Bhattacharya (1981) and Chaturvedi and Rani (1998), respectively. Inferences have been drawn for $\rho(t)$ and $P$ for some families of lifetime distributions by Chaturvedi and Rani (1997), Chaturvedi and Tomer (2003), Chaturvedi and Singh (2006, 2008), Chaturvedi and Kumari (2015) and Chaturvedi and Malhotra (2016, 2917). Chaturvedi and Tomer (2002) derived UMVUE for $\rho(t)$ and $P$ for negative binomial distribution. For exponentiated Weibull and Lomax distributions, the inferential procedures are available in Chaturvedi and Pathak (2012, 2013, 2014). Chaturvedi and Vyas (2017) developed estimation and testing procedures for the reliability functions of exponentiated distributions under Type I and Type II censoring.

Chandler (1952) introduced the concept of records as a statistic of successive extremes from a sequence of independent and identically distributed rvs. This theory is largely based on the theory of order statistics and is especially closely related to extreme order statistics. Record values and the associated statistics are of particular interest in the areas of climatology, sports, traffic, medicine, economics etc. A large number of record data saved for a long time motivated the development of several mathematical models reflecting the corresponding record processes and forecasting the future record results. Several inferential procedures for the parameters of different distributions, based on record data, have been developed by Glick (1978), Nagaraja (1988a,1988b), Balakrishan et al. (1995), Arnold et al. (1992), Habibi et al. (2006), Arashi and Emadi (2008), Razmkhah and Ahmadi (2011), Belaghi et al. (2015) and others.

The rest of the paper is organised as follows. In Section 2, we discuss the generalized inverted family of distributions proposed by Potdar and Shirke (2013) who introduced a shape parameter to the scale family of distributions. In Section 3, we develop point estimation procedures based on records when the scale parameter is known and also discuss the case when both the shape and scale parameters are unknown. As far as point estimation is concerned, we derive UMVUES and MLES. A new technique of obtaining these estimators is developed, in which first of all the estimators of powers of parameter are obtained. These estimators are used to obtain estimators of $\rho(t)$. Using the derivatives of the estimators of $\rho(t)$, the estimators of sampled probability density function $(p d f)$, at a specified point, are obtained which are subsequently used to obtain estimators of $P$. The estimators of $P$ are derived for the cases when $X$ and $Y$ belong to the same and different families of distributions. In Section 4, asymptotic confidence intervals for scale and shape parameters and reliability function are constructed and in Section 5, testing procedures are developed for various parametric functions. In Section 6, we present numerical findings and illustrate a real example.

\section{The Generalized Inverted Scale Family of Distributions}

Let $Y$ be a $r v$ having distribution belonging to a scale family of distributions with cumulative distribution function $(c d f) G$, probability density function $(p d f) g$ and scale parameter $\lambda$. Potdar and Shirke (2013) generalized this family by introducing a shape parameter $\alpha$ to obtain a generalized scale family of distributions. Let $X=\frac{1}{Y}$, then distribution of $X$ belongs to generalized inverted scale family of distributions. The $p d f$ and $c d f$ of the generalized inverted scale family of distributions are respectively given as:

$$
\begin{aligned}
& f_{X}(x ; \lambda, \alpha)=\frac{\alpha}{\lambda x^{2}} g\left(\frac{1}{\lambda x}\right)\left[G\left(\frac{1}{\lambda x}\right)\right]^{\alpha-1} ; \quad x>0, \lambda>0, \alpha>0 \\
& F_{X}(x ; \lambda, \alpha)=1-\left[G\left(\frac{1}{\lambda x}\right)\right]^{\alpha} ; \quad x>0, \lambda>0, \alpha>0
\end{aligned}
$$

We obtain the model in equation (2.1) by differentiating $F_{X}(x ; \lambda, \alpha)$ in (2.2) with respect to $x$. Some of the members of the family of distributions in (2.1) are generalized inverted exponential distribution (GIED), generalized inverted half-logistic distribution (GIHD), generalized inverted Rayleigh distribution (GIRD) and so on. The following Figure 1 shows the pdf plot of the generalized inverted scale family of distributions. 

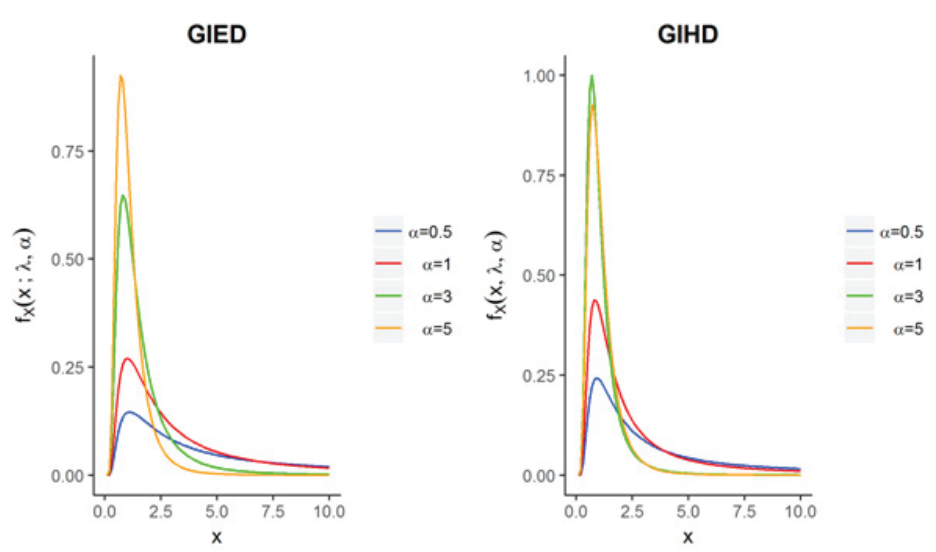

Figure 1. The pdf plot of some members of Generalized Inverted Scale Family of Distributions for different values of the shape parameter $\alpha$.

\section{Point Estimation Procedures}

Let $X_{1}, X_{2}, \ldots$ be an infinite sequence of independent and identically distributed (iid) rvs from (2.1). An observation $X_{j}$ will be called an upper record value (or simply a record) if its value exceeds than all the previous observations up to time $j$. Thus $X_{j}$ is a record if $X_{j}>X_{i}$ for every $i<j$.

The record time sequence $\left\{T_{n}, n \geq 0\right\}$ is a sequence of all time points when an observation is marked as a record and is mathematically defined as:

$$
\begin{cases}T_{0}=1 ; & \text { with probability } 1 \\ T_{n}=\min \left\{j: X_{j}>X_{T_{n-1}}\right\} ; & n \geq 1\end{cases}
$$

The record value sequence $\left\{R_{n}\right\}$ is a sequence of all observations marked as records and is mathematically defined as:

$$
R_{n}=X_{T_{n}} ; \quad n=0,1,2, \ldots
$$

We can rewrite (2.1) as follows:

$$
f_{X}(x ; \lambda, \alpha)=\frac{\alpha g\left(\frac{1}{\lambda x}\right)}{\left(\lambda x^{2} G\left(\frac{1}{\lambda x}\right)\right)} \exp \left\{-\alpha \log \left(\frac{1}{G\left(\frac{1}{\lambda x}\right)}\right)\right\} ; \quad x>0, \lambda>0, \alpha>0
$$

The likelihood function of the first $n+1$ upper record values $R_{0}, R_{1}, R_{2}, \ldots, R_{n}$ is:

$$
L\left(\alpha \mid R_{0}, R_{1}, R_{2}, \ldots, R_{n}\right)=f_{X}\left(R_{n} ; \lambda, \alpha\right) \prod_{i=0}^{n-1} \frac{f_{X}\left(R_{i} ; \lambda, \alpha\right)}{1-F_{X}\left(R_{i} ; \lambda, \alpha\right)}
$$

It is easy to see that

$$
L\left(\alpha \mid R_{0}, R_{1}, R_{2}, \ldots, R_{n}\right)=\left(\frac{\alpha}{\lambda}\right)^{n+1} \exp \left(-\alpha \log \left(\frac{1}{G\left(\frac{1}{\lambda R_{n}}\right)}\right)\right) \prod_{i=0}^{n} \frac{g\left(\frac{1}{\lambda R_{i}}\right)}{R_{i}^{2} G\left(\frac{1}{\lambda R_{i}}\right)}
$$

The following theorem provides UMVUE of powers of $\alpha$. This estimator will be utilized to obtain the UMVUE of reliability functions. For simplicity, we define:

$$
U(x)=\log \left(\frac{1}{G\left(\frac{1}{\lambda x}\right)}\right)
$$




\section{Theorem 1}

For $q \in(-\infty, \infty), q \neq 0$, the UMVUE of $\alpha^{q}$ is given by:

$$
\tilde{\alpha}^{q}= \begin{cases}\left\{\frac{\Gamma(n+1)}{\Gamma(n-q+1)}\right\}\left(U\left(R_{n}\right)\right)^{-q} ; & n>q-1 \\ 0 ; & \text { otherwise }\end{cases}
$$

Proof

It follows from (3.1) and factorisation theorem [see Rohtagi and Saleh (2012, p.361)] that $U\left(R_{n}\right)$ is a sufficient statistic for $\alpha$ and the $p d f$ of $U\left(R_{n}\right)$ is:

$$
h\left(U\left(R_{n}\right) \mid \alpha\right)=\frac{\alpha^{n+1} U\left(R_{n}\right)^{n}}{\Gamma(n+1)} \exp \left(-\alpha U\left(R_{n}\right)\right) ; U\left(R_{n}\right) \geq 0
$$

From (3.2), since the distribution of $U\left(R_{n}\right)$ belongs to exponential family, it is also complete [see Rohtagi and Saleh (2012, p.367)]. The result now follows from (3.2) that

$$
E\left[U\left(R_{n}\right)^{-q}\right]=\left\{\frac{\Gamma(n-q+1)}{\Gamma(n+1)}\right\} \alpha^{q}
$$

In the following theorem, we obtain UMVUE of the reliability function.

\section{Theorem 2}

The UMVUE of the reliability function is

$$
\tilde{\rho}(t)= \begin{cases}{\left[1-\frac{U(t)}{U\left(R_{n}\right)}\right]^{n} ;} & U(t)<U\left(R_{n}\right) \\ 0 ; & \text { otherwise }\end{cases}
$$

Proof

It is easy to see that

$$
\begin{aligned}
\rho(t) & =\exp \{-\alpha U(t)\} \\
& =\sum_{i=0}^{\infty} \frac{(-1)^{i}}{i !}\{\alpha U(t)\}^{i}
\end{aligned}
$$

Applying Theorem 1, it follows from (3.3) that

$$
\begin{aligned}
\tilde{\rho}(t) & =\sum_{i=0}^{\infty} \frac{(-1)^{i}}{i !}\{U(t)\}^{i} \tilde{\alpha}^{i} \\
& =\sum_{i=0}^{n}(-1)^{i}\left(\begin{array}{c}
n \\
i
\end{array}\right)\left\{\frac{U(t)}{U\left(R_{n}\right)}\right\}^{i}
\end{aligned}
$$

and the theorem follows.

The following corollary provides UMVUE of the sampled $p d f$. This estimator is derived with the help of Theorem 2.

Corollary 1

The UMVUE of the sampled $p d f(2.1)$ at a specified point $x$ is

$$
\tilde{f}_{X}(x ; \lambda, \alpha)= \begin{cases}\frac{n g\left(\frac{1}{\lambda x}\right)}{\lambda x^{2} U\left(R_{n}\right) G\left(\frac{1}{\lambda x}\right)}\left[1-\frac{U(x)}{U\left(R_{n}\right)}\right]^{n-1} ; & U(x)<U\left(R_{n}\right) \\ 0 ; & \text { otherwise }\end{cases}
$$




\section{Proof}

We note that the expectation of $\int_{t}^{\infty} \tilde{f}_{X}(x ; \lambda, \alpha) d x$ with respect to $R_{n}$ is $\rho(t)$. Hence, $\tilde{\rho}(t)=\int_{t}^{\infty} \tilde{f}_{X}(x ; \lambda, \alpha) d x$.

The result follows from Theorem 2.

In the following theorem, we obtain expression for the variance of $\tilde{\rho}(t)$, which will be needed to study its efficiency.

\section{Theorem 3}

The variance of $\tilde{\rho}(t)$ is given by:

$$
\begin{aligned}
\operatorname{Var}\{\tilde{\rho}(t)\}= & \frac{1}{n !}\{\alpha U(t)\}^{(n+1)} \exp \{-\alpha U(t)\}\left[\frac{a_{n}}{\alpha U(t)}-a_{n-1} \exp \{\alpha U(t)\} E_{i}(-\alpha U(t))\right. \\
& +\sum_{i=0}^{n-2} a_{i}\left\{\sum_{m=1}^{n-i-1} \frac{(m-1) !}{(n-i-1) !}(-\alpha U(t))^{n-i-m-1}\right. \\
& \left.-\frac{1}{(n-i-1) !}(-\alpha U(t))^{n-i-1} \exp (\alpha U(t)) E_{i}(-\alpha U(t))\right\} \\
& \left.+\sum_{i=n+1}^{2 n} a_{i}(i-n) !\left(\frac{1}{(\alpha U(t))}\right)^{i-n+1} \sum_{r=0}^{i-n} \frac{1}{r !}(\alpha U(t))^{r}\right] \\
& -\exp \{-2 \alpha U(t)\}
\end{aligned}
$$

where $a_{i}=(-1)^{i}\left(\begin{array}{c}2 n \\ i\end{array}\right)$ and $-E_{i}(-x)=\int_{x}^{\infty} \frac{e^{-u}}{u} d u$.

Proof

Using (3.2) and Theorem 2,

$$
\begin{aligned}
E\left\{\tilde{\rho}(t)^{2}\right\} & =\frac{\alpha^{n+1}}{\Gamma(n+1)} \int_{U(t)}^{\infty}\left[1-\frac{U(t)}{U\left(R_{n}\right)}\right]^{2 n}\left\{U\left(R_{n}\right)\right\}^{n} \exp \left\{-\alpha U\left(R_{n}\right)\right\} d U\left(R_{n}\right) \\
& =\frac{1}{(\Gamma(n+1))}(\alpha U(t))^{n+1} \exp (-\alpha U(t)) \int_{0}^{\infty} \frac{z^{2 n}}{(1+z)^{n}} \exp (-z \alpha U(t)) d z \\
& =\frac{1}{(\Gamma(n+1))}(\alpha U(t))^{n+1} \exp (-\alpha U(t)) I, \quad \text { (say) }
\end{aligned}
$$

where

$$
\begin{aligned}
I= & \sum_{i=0}^{n} a_{i} \int_{0}^{\infty} \frac{1}{(z+1)^{n-i}} \exp (-z \alpha U(t)) d z \\
& +\sum_{i=n+1}^{2 n} a_{i} \int_{0}^{\infty}(z+1)^{i-n} \exp (-z \alpha U(t)) d z
\end{aligned}
$$

Using a result of Erdélyi (1954) that

$$
\int_{0}^{\infty} \frac{(\exp ?(-u p))}{(u+a)^{n}} d u=\sum_{m=1}^{n-1} \frac{(m-1) !(-p)^{n-m-1}}{(n-1) ! a^{m}}-\frac{(-p)^{n-1}}{(n-1) !} \exp ?(a p) E_{i}(-a p)
$$


we have

$$
\begin{aligned}
\int_{0}^{\infty} & \frac{1}{(z+1)^{n-i}} \exp (-z \alpha U(t)) d z \\
= & \sum_{m=1}^{n-i-1} \frac{(m-1) !}{(n-i-1) !}(-\alpha U(t))^{n-i-m-1} \\
& -\frac{1}{(n-i-1) !}(-\alpha U(t))^{n-i-1} \exp (\alpha U(t)) E_{i}(-\alpha U(t)), \quad i=0,1,2, \ldots, n-2
\end{aligned}
$$

Furthermore,

$$
\begin{aligned}
\int_{0}^{\infty} \frac{1}{(1+z)} \exp (-z \alpha U(t)) d z & =\exp (\alpha U(t)) \int_{0}^{\infty} \frac{1}{(z+1)} \exp (-\alpha U(t)(z+1)) d z \\
& =\exp (\alpha U(t)) \int_{(\alpha U(t))}^{\infty} \frac{e^{-y}}{y} d y=-\exp (\alpha U(t)) E_{i}(-\alpha U(t)) .
\end{aligned}
$$

We have

$$
\int_{0}^{\infty} \exp (-z \alpha U(t)) d u=\left(\frac{1}{\alpha U(t)}\right)
$$

Finally,

$$
\begin{aligned}
\int_{0}^{\infty}(1+z)^{i-n} \exp (-z \alpha U(t)) d z & =\sum_{r=0}^{i-n}\left(\begin{array}{c}
i-n \\
r
\end{array}\right) \int_{0}^{\infty} z^{i-n-r} \exp ?(-z \alpha U(t)) d z \\
& =\sum_{r=0}^{i-n}\left(\begin{array}{c}
i-n \\
r
\end{array}\right)\left\{\frac{1}{\alpha U(t)}\right\}^{i-n-r+1} \Gamma(i-n-r+1)
\end{aligned}
$$

The theorem now follows on making substitutions from (3.7), (3.8), (3.9) and (3.10) in (3.6) and then using (3.5).

\section{Theorem 4}

The MLE of $\rho(t)$ is given by:

$$
\hat{\rho}(t)=\exp \left\{\frac{-(n+1) U(t)}{U\left(R_{n}\right)}\right\}
$$

Proof

It can be easily seen from (3.1) that the MLE of $\alpha$ is $\hat{\alpha}=\frac{(n+1)}{U\left(R_{n}\right)}$. The theorem now follows from invariance property of MLE.

In the following corollary, we obtain the MLE of sampled pdf with the help of Theorem 4. This will be used to obtain MLE of $P$.

Corollary 2

The MLE of $f_{X}(x ; \lambda, \alpha)$ at a specified point $x$ is

$$
\hat{f}_{X}(x ; \lambda, \alpha)=\frac{(n+1) g\left(\frac{1}{\lambda x}\right)}{\lambda x^{2} U\left(R_{n}\right) G\left(\frac{1}{\lambda x}\right)} \exp \left\{\frac{-(n+1) U(x)}{U\left(R_{n}\right)}\right\} ; \quad x>0, \lambda>0, \alpha>0
$$

Proof

The result follows from Theorem 4 on using the fact that $\hat{f}_{X}(x ; \lambda, \alpha)=-\frac{d}{d t} \hat{\rho}(t)$. 
In the following theorem, we obtain the expression for variance of $\hat{\rho}(t)$.

Theorem 5

The variance of $\hat{\rho}(t)$ is given by:

$$
\begin{aligned}
\operatorname{Var}\{\hat{\rho}(t)\}= & \frac{2}{n !}\{2(n+1) \alpha U(t)\}^{\frac{n+1}{2}} K_{n+1}(2 \sqrt{2(n+1) \alpha U(t)}) \\
& -\left[\frac{2}{n !}\{(n+1) \alpha U(t)\}^{\frac{n+1}{2}} K_{n+1}(2 \sqrt{(n+1) \alpha U(t)})\right]^{2}
\end{aligned}
$$

where $K_{r}(\cdot)$ is modified Bessel function of second kind of order $r$.

Proof

Using (3.2) and Theorem 4, we have

$$
\begin{aligned}
E\{\hat{\rho}(t)\} & =\frac{\alpha^{n+1}}{\Gamma(n+1)} \int_{0}^{\infty} \exp \left[-\left\{\alpha U\left(R_{n}\right)+\frac{(n+1) U(t)}{U\left(R_{n}\right)}\right\}\right]\left\{U\left(R_{n}\right)\right\}^{n} d U\left(R_{n}\right) \\
& =\frac{1}{\Gamma(n+1)} \int_{0}^{\infty} \exp \left[-\left\{y+\frac{(n+1) \alpha U(t)}{y}\right\}\right] y^{n} d y
\end{aligned}
$$

Applying a result of Watson (1952) that

$$
\int_{0}^{\infty} u^{-m} \exp \left\{-\left(a u+\frac{b}{u}\right)\right\} d u=2\left(\frac{a}{b}\right)^{\frac{m-1}{2}} K_{m-1}(2 \sqrt{a b})
$$

[it is to be noted that $K_{-m}(\cdot)=K_{m}(\cdot)$ for $m=0,1,2, \ldots$ ], we obtain from (3.11) that

$$
E\{\hat{\rho}(t)\}=\frac{2}{n !}\{(n+1) \alpha U(t)\}^{\frac{n+1}{2}} K_{n+1}(2 \sqrt{(n+1) \alpha U(t)})
$$

Similarly, we can obtain the expression for $E\left\{\hat{\rho}(t)^{2}\right\}$ and the result follows.

Let $X$ and $Y$ be two independent rvs following the generalized inverted scale families of distributions $f_{X}\left(x ; \lambda_{1}, \alpha_{1}\right)$ and $f_{Y}\left(y ; \lambda_{2}, \alpha_{2}\right)$ respectively. We consider the case when $X$ and $Y$ belong to different families of distributions, i.e.

$$
f_{X}\left(x ; \lambda_{1}, \alpha_{1}\right)=\frac{\alpha_{1} g\left(\frac{1}{\lambda_{1} x}\right)}{\lambda_{1} x^{2} G\left(\frac{1}{\lambda_{1} x}\right)} \exp \left\{-\alpha_{1} \log \left(\frac{1}{G\left(\frac{1}{\lambda_{1} x}\right)}\right)\right\} ; \quad x>0, \lambda_{1}>0, \alpha_{1}>0
$$

and

$$
f_{Y}\left(y ; \lambda_{2}, \alpha_{2}\right)=\frac{\alpha_{2} h\left(\frac{1}{\lambda_{2} y}\right)}{\lambda_{2} y^{2} H\left(\frac{1}{\lambda_{2} y}\right)} \exp \left\{-\alpha_{2} \log \left(\frac{1}{H\left(\frac{1}{\lambda_{2} y}\right)}\right)\right\} ; \quad y>0, \lambda_{2}>0, \alpha_{2}>0
$$

Let $\left\{R_{n}\right\}$ and $\left\{R_{m}^{*}\right\}$ be the record value sequences for $X$ 's and $Y$ 's respectively. For simplicity, we define:

$$
\begin{aligned}
& U(x)=\log \left(\frac{1}{G\left(\frac{1}{\lambda_{1} x}\right)}\right) \\
& V(x)=\log \left(\frac{1}{H\left(\frac{1}{\lambda_{2} y}\right)}\right)
\end{aligned}
$$


The following theorem provides the UMVUE of $P$ when $X$ and $Y$ belong to different families of distributions.

\section{Theorem 6}

The UMVUE of $P$ is given by

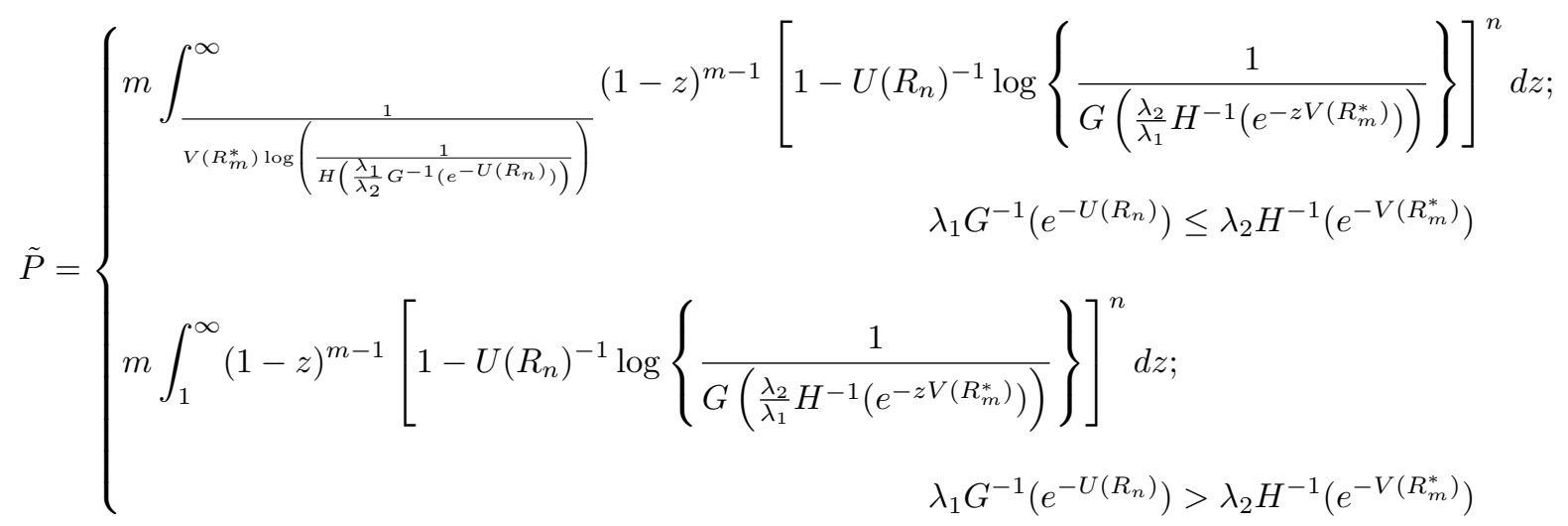

Proof

It follows from Corollary 1 that the UMVUES of $f_{X}\left(x ; \lambda_{1}, \alpha_{1}\right)$ and $f_{Y}\left(y ; \lambda_{2}, \alpha_{2}\right)$ at specified points $x$ and $y$ are respectively:

$$
\tilde{f}_{X}\left(x ; \lambda_{1}, \alpha_{1}\right)= \begin{cases}\frac{n g\left(\frac{1}{\lambda_{1} x}\right)}{\lambda_{1} x^{2} U\left(R_{n}\right) G\left(\frac{1}{\lambda_{1} x}\right)}\left[1-\frac{U(x)}{U\left(R_{n}\right)}\right]^{n-1} ; & U(x)<U\left(R_{n}\right) \\ 0 ; & \text { otherwise }\end{cases}
$$

and

$$
\tilde{f}_{Y}\left(y ; \lambda_{2}, \alpha_{2}\right)= \begin{cases}\frac{m h\left(\frac{1}{\lambda_{2} y}\right)}{\lambda_{2} y^{2} V\left(R_{m}^{*}\right) H\left(\frac{1}{\lambda_{2} y}\right)}\left[1-\frac{V(y)}{V\left(R_{m}^{*}\right)}\right]^{n-1} ; & V(y)<V\left(R_{m}^{*}\right) \\ 0 ; & \text { otherwise }\end{cases}
$$

From the arguments similar to those used in the proof of Corollary 1,

$$
\begin{aligned}
\tilde{P} & =\int_{y=0}^{\infty} \int_{x=y}^{\infty} \tilde{f}_{X}\left(x ; \lambda_{1}, \alpha_{1}\right) \tilde{f}_{Y}\left(y ; \lambda_{2}, \alpha_{2}\right) d x d y \\
& =\int_{y=0}^{\infty} \tilde{\rho}_{X}(y)\left\{-\frac{d}{d y} \tilde{\rho}_{Y}(y)\right\} d y \\
& =m \int_{\max \left[\lambda_{1} G^{-1}\left(e^{-U\left(R_{n}\right)}\right), \lambda_{2} H^{-1}\left(e^{\left.\left.\left(-V\left(R_{m}^{*}\right)\right)\right)\right]}\right.\right.}^{\infty}\left[1-\frac{U(y)}{U\left(R_{n}\right)}\right]^{n}\left\{\frac{h\left(\frac{1}{\lambda_{2} y}\right)}{\lambda_{2} y^{2} V\left(R_{m}^{*}\right) H\left(\frac{1}{\lambda_{2} y}\right)}\right\}\left[1-\frac{V(y)}{V\left(R_{m}^{*}\right)}\right]^{m-1} d y
\end{aligned}
$$

The theorem now follows on considering the two cases and putting $\frac{V(y)}{V\left(R_{m}^{*}\right)}=z$.

In the following theorem, we obtain the UMVUE of $P$ when $X$ and $Y$ belong to same families of distributions. 
Theorem 7

When $X$ and $Y$ belong to same families of distributions and $\lambda_{1}=\lambda_{2}$

$$
\tilde{P}= \begin{cases}1-m \sum_{i=0}^{m-1}(-1)^{i}\left(\begin{array}{c}
m-1 \\
i
\end{array}\right)\left\{\frac{U\left(R_{n}\right)}{U\left(R_{m}^{*}\right)}\right\}^{i+1} B(i+1, n+1) ; & U\left(R_{n}\right)<U\left(R_{m}^{*}\right) \\
1-m \sum_{i=0}^{n}(-1)^{i}\left(\begin{array}{c}
n \\
i
\end{array}\right)\left\{\frac{U\left(R_{m}^{*}\right)}{U\left(R_{n}\right)}\right\}^{i} B(i+1, m) ; & U\left(R_{m}^{*}\right)<U\left(R_{n}\right)\end{cases}
$$

Proof

Taking $G(\cdot)=H(\cdot)$ in Theorem 6 , then for $U\left(R_{n}\right)<U\left(R_{m}^{*}\right)$

$$
\begin{aligned}
\tilde{P} & =m \int_{\frac{U\left(R_{n}\right)}{U\left(R_{m}^{*}\right)}}^{\infty}(1-z)^{m-1}\left\{1-\frac{z U\left(R_{m}^{*}\right)}{U\left(R_{n}\right)}\right\}^{n} d z \\
& =1-m\left\{\frac{U\left(R_{n}\right)}{U\left(R_{m}^{*}\right)}\right\} \int_{0}^{1}\left\{1-\frac{w U\left(R_{n}\right)}{U\left(R_{m}^{*}\right)}\right\}^{m-1}(1-w)^{n} d w \\
& =1-m \sum_{i=0}^{m-1}(-1)^{i}\left(\begin{array}{c}
m-1 \\
i
\end{array}\right)\left\{\frac{U\left(R_{n}\right)}{U\left(R_{m}^{*}\right)}\right\}^{i+1} \int_{0}^{1} w^{i}(1-w)^{n} d w
\end{aligned}
$$

and the first assertion follows. Similarly, we can prove the second assertion.

The following theorem provides the MLE of $P$ when $X$ and $Y$ belong to different families of distributions.

Theorem 8

The MLE of $P$ when $X$ and $Y$ belong to different families of distributions, is

$$
\hat{P}=\int_{0}^{\infty} e^{-z} \exp \left\{\frac{-(n+1)}{U\left(R_{n}\right)} \log \left(\frac{1}{G\left(\frac{\lambda_{2}}{\lambda_{1}} H^{-1}\left(e^{\frac{-z V\left(R_{m}^{*}\right)}{m+1}}\right)\right)}\right)\right\} d z
$$

Proof

We have,

$$
\begin{aligned}
\hat{P} & =\int_{y=0}^{\infty} \int_{x=y}^{\infty} \hat{f}_{X}\left(x ; \lambda_{1}, \alpha_{1}\right) \hat{f}_{Y}\left(y ; \lambda_{2}, \alpha_{2}\right) d x d y \\
& =\int_{y=0}^{\infty} \hat{\rho}_{X}(y) \hat{f}_{Y}\left(y ; \lambda_{2}, \hat{\alpha}_{2}\right) d y \\
& =\int_{y=0}^{\infty} \exp \left\{\frac{-(n+1) U(y)}{U\left(R_{n}\right)}\right\}\left\{\frac{(m+1) h\left(\frac{1}{\lambda_{2} y}\right)}{\lambda_{2} y^{2} V\left(R_{m}^{*}\right) H\left(\frac{1}{\lambda_{2} y}\right)}\right\} \exp \left\{\frac{-(m+1) V(y)}{V\left(R_{m}^{*}\right)}\right\} d y
\end{aligned}
$$

The result now follows on putting $\left\{\frac{(m+1) V(y)}{V\left(R_{m}^{*}\right)}\right\}=z$.

The following theorem provides MLE of $P$ when $X$ and $Y$ belong to same families of distributions. The result follows from Theorem 8 .

Theorem 9

When $X$ and $Y$ belong to same families of distributions and $\lambda_{1}=\lambda_{2}$, the MLE of $P$ is given by

$$
\hat{P}=\frac{(m+1) U\left(R_{n}\right)}{(m+1) U\left(R_{n}\right)+(n+1) U\left(R_{m}^{*}\right)}
$$


Now we consider the case when both the parameters $\alpha$ and $\lambda$ are unknown. From (3.1), the log-likelihood function is given as:

$$
\begin{aligned}
l(\alpha, \lambda) & =L\left(\alpha, \lambda \mid R_{0}, R_{1}, R_{2}, \ldots, R_{n}\right) \\
& =(n+1) \log (\alpha)-(n+1) \log (\lambda)-\alpha U\left(R_{n}\right)+\sum_{i=0}^{n} \log \left(g\left(\frac{1}{\lambda R_{i}}\right)\right)-2 \log \left(R_{i}\right)-\log \left(G\left(\frac{1}{\left(\lambda R_{i}\right)}\right)\right)
\end{aligned}
$$

where

$$
U(x)=\log \left(\frac{1}{G\left(\frac{1}{\lambda x}\right)}\right)
$$

The MLES of $\alpha$ and $\lambda$ are the solutions of the two simultaneous equations given below:

$$
\frac{n+1}{\alpha}-U\left(R_{n}\right)=0
$$

and

$$
\frac{-(n+1)}{\lambda}-\frac{1}{\lambda^{2}} \sum_{i=0}^{n} \frac{g^{\prime}\left(\frac{1}{\lambda R_{i}}\right)}{R_{i} g\left(\frac{1}{\lambda R_{i}}\right)}+\frac{1}{\lambda^{2}} \sum_{i=0}^{n} \frac{g\left(\frac{1}{\lambda R_{i}}\right)}{R_{i} G\left(\frac{1}{\lambda R_{i}}\right)}-\alpha \frac{g\left(\frac{1}{\lambda R_{n}}\right)}{\lambda^{2} R_{n} G\left(\frac{1}{\lambda R_{n}}\right)}=0
$$

From (3.13), we get

$$
\hat{\alpha}=\frac{n+1}{\log \left(\frac{1}{G\left(\frac{1}{\lambda R_{n}}\right)}\right)}
$$

where $\hat{\alpha}$ and $\hat{\lambda}$ are the MLES of $\alpha$ and $\lambda$ respectively.

Since these non-linear equation does not have a closed form solution, therefore we apply Newton Raphson algorithm to compute MLE of $\lambda$. Using this values of $\hat{\lambda}$, we can compute $\hat{\alpha}$ from (3.15).

It is to be noted that from Theorem 4, Theorem 8 and invariance property of MLE, the MLE of $\rho(t)$ is given as:

$$
\hat{\rho}(t)=\exp \left\{\frac{-(n+1) U(t)}{U\left(R_{n}\right)}\right\}
$$

where $U(x)=\log \left(\frac{1}{G\left(\frac{1}{\lambda x}\right)}\right), \hat{\lambda}$ is the MLE of $\lambda$. Whereas the MLE of $P$ when $X$ and $Y$ belong to different family of distribution is given by:

$$
\hat{P}=\int_{0}^{\infty} e^{-z} \exp \left\{\frac{-(n+1)}{U\left(R_{n}\right)} \log \left(\frac{1}{G\left(\frac{\hat{\lambda}_{2}}{\hat{\lambda}_{1}} H^{-1}\left(e^{-z V\left(R_{m}^{*}\right)} m+1\right)\right)}\right)\right\} d z
$$

where $U(x)=\log \left(\frac{1}{G\left(\frac{1}{\lambda_{1} x}\right)}\right), V(x)=\log \left(\frac{1}{H\left(\frac{1}{\lambda_{2} x}\right)}\right)$ and $\hat{\lambda}_{1}$ and $\hat{\lambda}_{2}$ are the MLES of $\lambda_{1}$ and $\lambda_{2}$ respectively. Similarly, the MLE of $P$ when $X$ and $Y$ belong to same family of distribution and $\lambda_{1}=\lambda_{2}$ can be derived from Theorem 9. 


\section{Confidence Intervals}

Now, Fisher information matrix of $\theta=(\alpha, \lambda)^{T}$ is:

$$
I(\theta)=-E\left[\begin{array}{cc}
\frac{\partial^{2} l}{\partial \alpha^{2}} & \frac{\partial^{2} l}{\partial \alpha \partial \lambda} \\
\frac{\partial^{2} l}{\partial \lambda \partial \alpha} & \frac{\partial^{2} l}{\partial \lambda^{2}}
\end{array}\right]
$$

where $\frac{\partial^{2} l}{\partial \alpha^{2}}=\frac{-(n+1)}{\alpha^{2}}, \frac{\partial^{2} l}{\partial \alpha \partial \lambda}=\frac{\partial^{2} l}{\partial \lambda \partial \alpha}=\frac{-g\left(\frac{1}{\lambda R_{n}}\right)}{\lambda^{2} R_{n} G\left(\frac{1}{\lambda R_{n}}\right)}$

$$
\begin{aligned}
\frac{\partial^{2} l}{\partial \lambda^{2}}= & \frac{n+1}{\lambda^{2}}+\frac{1}{\lambda^{4}} \sum_{i=0}^{n} \frac{\left\{g\left(\frac{1}{\lambda R_{i}}\right) g^{\prime \prime}\left(\frac{1}{\lambda R_{i}}\right)-\left(g^{\prime}\left(\frac{1}{\lambda R_{i}}\right)\right)^{2}+2 \lambda R_{i} g\left(\frac{1}{\lambda R_{i}}\right) g^{\prime}\left(\frac{1}{\lambda R_{i}}\right)\right\}}{\left\{R_{i} g\left(\frac{1}{\lambda R_{i}}\right)\right\}^{2}} \\
& -\frac{1}{\lambda^{4}} \sum_{i=0}^{n} \frac{\left\{G\left(\frac{1}{\lambda R_{i}}\right) g^{\prime}\left(\frac{1}{\lambda R_{i}}\right)-\left(g\left(\frac{1}{\lambda R_{i}}\right)\right)^{2}+2 \lambda R_{i} g\left(\frac{1}{\lambda R_{i}}\right) G\left(\frac{1}{\lambda R_{i}}\right)\right\}}{\left\{R_{i} G\left(\frac{1}{\lambda R_{i}}\right)\right\}^{2}} \\
& +\frac{\alpha}{\lambda^{4}} \frac{\left\{G\left(\frac{1}{\lambda R_{n}}\right) g^{\prime}\left(\frac{1}{\lambda R_{n}}\right)-\left(g\left(\frac{1}{\lambda R_{n}}\right)\right)^{2}+2 \lambda R_{n} g\left(\frac{1}{\lambda R_{n}}\right) G\left(\frac{1}{\lambda R_{n}}\right)\right\}}{\left\{R_{n} G\left(\frac{1}{\lambda R_{n}}\right)\right\}^{2}}
\end{aligned}
$$

where $g^{\prime}(\cdot)=\frac{d}{d \lambda} g(\cdot)$ and $g^{\prime \prime}(\cdot)=\frac{d}{\lambda} g^{\prime}(\cdot)$.

Since it is a complicated task to obtain the expectation of the above expressions, therefore we use observed Fisher information matrix which is obtained by dropping the expectation sign. The asymptotic variance-covariance matrix of the MLES is the inverse of $I(\hat{\theta})$. After obtaining the inverse matrix, we get variance of $\hat{\alpha}$ and $\hat{\lambda}$. We use these values to construct confidence intervals of $\alpha$ and $\lambda$ respectively.

Assuming asymptotic normality of the MLES, CIs for $\alpha$ and $\lambda$ are constructed. Let $\hat{\sigma}^{2}(\hat{\alpha})$ and $\hat{\sigma}^{2}(\hat{\lambda})$ be the estimated variances of $\hat{\alpha}$ and $\hat{\lambda}$ respectively. Then $100(1-\varepsilon) \%$ asymptotic CIs for $\alpha$ and $\lambda$ are respectively given by:

$$
\left(\hat{\alpha}-Z_{\frac{\varepsilon}{2}} \hat{\sigma}(\hat{\alpha}), \hat{\alpha}+Z_{\frac{\varepsilon}{2}} \hat{\sigma}(\hat{\alpha})\right) \text { and }\left(\hat{\lambda}-Z_{\frac{\varepsilon}{2}} \hat{\sigma}(\hat{\lambda}), \hat{\lambda}+Z_{\frac{\varepsilon}{2}} \hat{\sigma}(\hat{\lambda})\right)
$$

where $Z_{\frac{\varepsilon}{2}}$ is the upper $100(1-\varepsilon)$ percentile point of standard normal distribution. Using this CI for $\alpha$ and $\lambda$, one can easily obtain the $100(1-\varepsilon) \%$ asymptotic $\mathrm{CI}$ for $\rho(t)$ as follows:

$$
\left(\left(G\left(\frac{1}{t\left(\hat{\lambda}+Z_{\frac{\varepsilon}{2}} \hat{\sigma}(\hat{\lambda})\right)}\right)\right)^{\hat{\alpha}+Z_{\frac{\varepsilon}{2}} \hat{\sigma}(\hat{\alpha})},\left(G\left(\frac{1}{t\left(\hat{\lambda}-Z_{\frac{\varepsilon}{2}} \hat{\sigma}(\hat{\lambda})\right)}\right)\right)^{\hat{\alpha}-Z_{\frac{\varepsilon}{2}} \hat{\sigma}(\hat{\alpha})}\right)
$$

Meeker and Escober (1998) reported that the asymptotic CI based on $\log ($ MLE) has better coverage probability. An approximate $100(1-\varepsilon) \%$ CI for $\log (\alpha)$ and $\log (\lambda)$ are:

$$
\left(\log (\hat{\alpha})-Z_{\frac{\varepsilon}{2}} \hat{\sigma}(\log (\hat{\alpha})), \log (\hat{\alpha})+Z_{\frac{\varepsilon}{2}} \hat{\sigma}(\log (\hat{\alpha}))\right)
$$

and

$$
\left(\log (\hat{\lambda})-Z_{\frac{\varepsilon}{2}} \hat{\sigma}(\log (\hat{\lambda})), \log (\hat{\lambda})+Z_{\frac{\varepsilon}{2}} \hat{\sigma}(\log (\hat{\lambda}))\right)
$$

where $\hat{\sigma}^{2}(\log (\hat{\alpha}))$ is the estimated variance of $\log (\alpha)$ and is approximated by $\hat{\sigma}^{2}(\log (\hat{\alpha}))=\frac{\hat{\sigma}^{2}(\hat{\alpha})}{\hat{\alpha}^{2}}$. Similarly, $\hat{\sigma}^{2}(\log (\hat{\lambda}))$ is the estimated variance of $\log (\lambda)$ and is approximated by $\hat{\sigma}^{2}(\log (\hat{\lambda}))=\frac{\hat{\sigma}^{2}(\hat{\lambda})}{\hat{\lambda}^{2}}$. Hence, approximate 
$100(1-\varepsilon) \%$ CI for $\alpha$ and $\lambda$ are:

$$
\left(\hat{\alpha} e^{-Z_{\frac{\varepsilon}{2}} \frac{\hat{\sigma}(\hat{\alpha})}{\hat{\alpha}}}, \hat{\alpha} e^{Z_{\frac{\varepsilon}{2}} \frac{\hat{\sigma}(\hat{\alpha})}{\hat{\alpha}}}\right) \quad \text { and }\left(\hat{\lambda} e^{-Z_{\frac{\varepsilon}{2}} \frac{\hat{\sigma}(\hat{\lambda})}{\hat{\lambda}}}, \hat{\lambda} e^{Z_{\frac{\varepsilon}{2}} \frac{\hat{\sigma}(\hat{\lambda})}{\hat{\lambda}}}\right)
$$

\section{Testing of Hypotheses}

Suppose, for known value of $\lambda$, we have to test the hypothesis $H_{0}: \alpha=\alpha_{0}$ against $H_{1}: \alpha \neq \alpha_{0}$. It follows from (3.1) that, under $H_{0}$,

$$
\sup _{\Theta_{0}} ?\left(\alpha \mid R_{0}, R_{1}, \ldots, R_{n}\right)=\left(\frac{\alpha_{0}}{\lambda}\right)^{n+1} \exp \left(-\alpha_{0} \log \left(1 / G\left(\frac{1}{\lambda R_{n}}\right)\right)\right) \prod_{i=0}^{n} \frac{g\left(\frac{1}{\lambda R_{i}}\right)}{R_{i}^{2} G\left(\frac{1}{\lambda R_{i}}\right)} ; \Theta_{0}=\left\{\alpha: \alpha=\alpha_{0}\right\}
$$

and

$$
\sup _{\Theta} L\left(\alpha \mid R_{0}, R_{1}, \ldots, R_{n}\right)=\left(\frac{n+1}{\lambda \log \left(\frac{1}{G\left(\frac{1}{\lambda R_{n}}\right)}\right)}\right)^{n+1} \exp (-(n+1)) \prod_{i=0}^{n} \frac{g\left(\frac{1}{\lambda R_{i}}\right)}{R_{i}^{2} G\left(\frac{1}{\lambda R_{i}}\right)} ; \Theta=\{\alpha: \alpha>0\}
$$

Denoting $\log \left(\frac{1}{G\left(\frac{1}{\lambda x}\right)}\right)$ by $U(x)$. The likelihood ratio (LR) is given by:

$$
\begin{aligned}
\Phi\left(R_{0}, R_{1}, \ldots, R_{n}\right) & =\frac{\sup _{\Theta_{0}} ? L\left(\alpha \mid R_{0}, R_{1}, \ldots, R_{n}\right)}{\sup _{\Theta} L\left(\alpha \mid R_{0}, R_{1}, \ldots, R_{n}\right)} \\
& =\left\{\frac{\alpha_{0} U\left(R_{n}\right)}{(n+1)}\right\}^{n+1} \exp \left\{-\alpha_{0} U\left(R_{n}\right)+(n+1)\right\}
\end{aligned}
$$

We note that the first term on the right hand side of (5.1) is monotonically increasing and the second term is monotonically decreasing in $U\left(R_{n}\right)$. It follows from (3.2) that $2 \alpha_{0} U\left(R_{n}\right) \sim \chi_{2(n+1)}^{2}$. Thus, the critical region is given by:

$$
\left\{0<U\left(R_{n}\right)<k_{0}\right\} \cup\left\{k_{0}^{\prime}<U\left(R_{n}\right)<\infty\right\}
$$

where $k_{0}$ and $k_{0}^{\prime}$ are obtained such that $k_{0}=\frac{\chi_{2(n+1)}^{2}\left(\frac{\varepsilon}{2}\right)}{2 \alpha_{0}}$ and $k_{0}^{\prime}=\frac{\chi_{2(n+1)}^{2}\left(1-\frac{\varepsilon}{2}\right)}{2 \alpha_{0}}$ where $\varepsilon$ is the level of significance.

An important hypothesis in life-testing experiments is $H_{0}: \alpha \leq \alpha_{0}$ against $H_{1}: \alpha>\alpha_{0}$. It follows from (3.1) that for $\alpha_{1}>\alpha_{2}$,

$$
\frac{L\left(\alpha_{1} \mid R_{0}, R_{1}, \ldots, R_{n}\right)}{L\left(\alpha_{2} \mid R_{0}, R_{1}, \ldots, R_{n}\right)}=\left(\frac{\alpha_{1}}{\alpha_{2}}\right)^{n+1} \exp \left\{\left(\alpha_{2}-\alpha_{1}\right) U\left(R_{n}\right)\right\}
$$

It follows from (5.2) that the family of distributions $f_{X}(x ; \lambda, \alpha)$ has monotone likelihood ratio in $U\left(R_{n}\right)$. Thus, the uniformly most powerful critical region for testing $H_{0}$ against $H_{1}$ is given by [see Lehmann $(1959, \mathrm{p} .88)$ ]

$$
\Phi\left(R_{0}, R_{1}, \ldots, R_{n}\right)= \begin{cases}1 ; & U\left(R_{n}\right) \leq k_{0}^{\prime \prime} \\ 0 ; & \text { otherwise }\end{cases}
$$

where $k_{0}^{\prime \prime}=\frac{\chi_{2(n+1)}^{2}(\varepsilon)}{2 \alpha_{0}}$.

It can be seen that when $X$ and $Y$ belong to same families of distributions and $\lambda_{1}=\lambda_{2}=\lambda, P=\frac{\alpha_{2}}{\alpha_{1}+\alpha_{2}}$. 
Suppose we want to test $H_{0}: P=P_{0}$ against $H_{1}: P \neq P_{0}$. It follows that $H_{0}$ is equivalent to $\alpha_{2}=k \alpha_{1}$ where $k=\frac{P_{0}}{1-P_{0}}$. Thus, $H_{0}: \alpha_{2}=k \alpha_{1}$ and $H_{1}: \alpha_{2} \neq k \alpha_{1}$.

It can be shown that, under $H_{0}$,

$$
\hat{\alpha}_{1}=\frac{n+m+2}{U\left(R_{n}\right)+k U\left(R_{m}^{*}\right)}
$$

and

$$
\hat{\alpha}_{2}=\frac{k(n+m+2)}{U\left(R_{n}\right)+k U\left(R_{m}^{*}\right)}
$$

The likelihood for observing $\alpha_{1}$ and $\alpha_{2}$ is

$$
\begin{aligned}
& L\left(\alpha_{1}, \alpha_{2} \mid R_{0}, R_{1}, \ldots, R_{n}, R_{0}^{*}, R_{1}^{*}, \ldots, R_{m}^{*}\right) \\
& \quad=\left(\frac{\alpha_{1}}{\lambda}\right)^{n+1}\left(\frac{\alpha_{2}}{\lambda}\right)^{m+1} \exp \left(-\left\{\alpha_{1} U\left(R_{n}\right)+\alpha_{2} U\left(R_{m}^{*}\right)\right\}\right) \prod_{i=0}^{n} \frac{g\left(\frac{1}{\lambda R_{i}}\right)}{R_{i}^{2} G\left(\frac{1}{\lambda R_{i}}\right)} \prod_{j=0}^{m} \frac{g\left(\frac{1}{\lambda R_{j}^{*}}\right)}{\left(R_{j}^{*}\right)^{2} G\left(\frac{1}{\lambda R_{j}^{*}}\right)}
\end{aligned}
$$

Thus, for a generic constant $C$,

$$
\begin{aligned}
& \sup _{\Theta_{0}} ? L\left(\alpha_{1}, \alpha_{2} \mid R_{0}, R_{1}, \ldots, R_{n}, R_{0}^{*}, R_{1}^{*}, \ldots, R_{m}^{*}\right) \\
& \quad=\frac{C}{\left\{U\left(R_{n}\right)+k U\left(R_{m}^{*}\right)\right\}^{n+m+2}} \exp \{-(n+m+2)\} ; \Theta_{0}=\left\{\alpha_{1}, \alpha_{2}: \alpha_{2}=k \alpha_{1}\right\}
\end{aligned}
$$

and

$$
\begin{aligned}
& \sup _{\Theta} L\left(\alpha_{1}, \alpha_{2} \mid R_{0}, R_{1}, \ldots, R_{n}, R_{0}^{*}, R_{1}^{*}, \ldots, R_{m}^{*}\right) \\
& \quad=\frac{C}{\left\{U\left(R_{n}\right)\right\}^{n+1}\left\{U\left(R_{m}^{*}\right)\right\}^{m+1}} \exp \{-(n+m+2)\} ; \quad \Theta=\left\{\alpha_{1}, \alpha_{2}: \alpha_{1}>0, \alpha_{2}>0\right\}
\end{aligned}
$$

From (5.3) and (5.4), the LR is:

$$
\Theta\left(R_{0}, R_{1}, \ldots, R_{n}, R_{0}^{*}, R_{1}^{*}, \ldots, R_{m}^{*}\right)=\frac{C\left\{\frac{U\left(R_{n}\right)}{U\left(R_{m}^{*}\right)}\right\}^{m+1}}{\left\{1+U\left(R_{n}\right) / k U\left(R_{m}^{*}\right)\right\}^{n+m+2}}
$$

Denoting by $F_{a, b}(\cdot)$, the $F$-Statistic with $(a, b)$ degrees of freedom and using the fact that $\frac{U\left(R_{n}\right)}{U\left(R_{m}^{*}\right)} \sim$ $\frac{(n+1) \alpha_{2}}{(m+1) \alpha_{1}} F_{2(n+1), 2(m+1)}$, the critical region is given by

$$
\left\{\frac{U\left(R_{n}\right)}{U\left(R_{m}^{*}\right)}<k_{2}\right\} \cup\left\{\frac{U\left(R_{n}\right)}{U\left(R_{m}^{*}\right)}>k_{2}^{\prime}\right\}
$$

where $k_{2}=\frac{k(n+1)}{(m+1)} F_{2(n+1), 2(m+1)}\left(\frac{\varepsilon}{2}\right)$ and $k_{2}^{\prime}=\frac{k(n+1)}{(m+1)} F_{2(n+1), 2(m+1)}\left(1-\frac{\varepsilon}{2}\right)$.

\section{Numerical Findings}

A simulation study is carried out to study the performance of MLES of $\alpha$ and $\lambda$ and compare the performance of UMVUE and MLE of $\alpha$ where we consider Generalized Inverted Exponential distribution (GIED). We compute bias and mean square errors of the estimators for comparison. Also, the length of asymptotic confidence intervals based on MLE and log-transformed MLE of $\alpha$ and $\lambda$ are compared. 
Simulation is carried out for $(\alpha, \lambda)=(0.5,0.5),(0.5,1),(1,0.5)$ and $(1,2)$ for $n=5,8,10$ and 12 . For each $n, 1000$ observations from gamma $(n+1, \alpha)$ were generated. Let us denote these observations by $Y_{i}$; $i=$ $1,2, \ldots, 1000$. Thus the average estimate of complete and sufficient statistic $U\left(R_{n}\right)$ is given by $U\left(R_{n}\right)=$ $\frac{1}{1000} \sum_{i=1}^{1000} Y_{i}$. Tables 1 to 4 show the bias and mean square errors of the MLES of $\alpha$ and $\lambda$ and UMVUE of $\alpha$. In Tables 5 to 8, the length of asymptotic confidence intervals based on MLE and log-transformed MLE of $\alpha$ and $\lambda$ at $95 \%$ and $90 \%$ level of significance are compared for different sample sizes $n$.

Table 1. When $\alpha=0.5$ and $\lambda=0.5$

\begin{tabular}{|c|c|c|c|c|c|c|c|c|}
\hline & \multicolumn{2}{|r|}{$\tilde{\alpha}$} & \multicolumn{3}{|c|}{$\hat{\alpha}$} & \multicolumn{3}{|c|}{$\hat{\lambda}$} \\
\hline$n$ & $\tilde{\alpha}$ & $M S E(\tilde{\alpha})$ & $\hat{\alpha}$ & $\operatorname{Bias}(\hat{\alpha})$ & $M S E(\hat{\alpha})$ & $\hat{\lambda}$ & $\operatorname{Bias}(\hat{\lambda})$ & $M S E(\hat{\lambda})$ \\
\hline 5 & 0.5642 & 0.0625 & 0.6770 & 0.1000 & 0.1000 & 0.4871 & -0.0128 & 0.5291 \\
\hline 8 & 0.6033 & 0.0357 & 0.6787 & 0.0625 & 0.0491 & 0.5112 & 0.0112 & 0.2185 \\
\hline 10 & 0.5971 & 0.0277 & 0.6568 & 0.0500 & 0.0361 & 1.0561 & 0.5561 & 0.3361 \\
\hline 12 & 0.6667 & 0.0227 & 0.7223 & 0.0416 & 0.0284 & 0.5216 & 0.0216 & 0.1232 \\
\hline
\end{tabular}

Table 2. When $\alpha=0.5$ and $\lambda=1$

\begin{tabular}{ccccccccc}
\hline & \multicolumn{2}{c}{$\tilde{\alpha}$} & $\hat{\alpha}$ & \multicolumn{3}{c}{$\hat{\lambda}$} \\
\hline$n$ & $\tilde{\alpha}$ & $M S E(\tilde{\alpha})$ & $\hat{\alpha}$ & $\operatorname{Bias}(\hat{\alpha})$ & $M S E(\hat{\alpha})$ & $\hat{\lambda}$ & $\operatorname{Bias}(\hat{\lambda})$ & $M S E(\hat{\lambda})$ \\
\hline 5 & 0.4634 & 0.0625 & 0.5561 & 0.1000 & 0.1000 & 1.1571 & 0.1571 & 0.8439 \\
8 & 0.6205 & 0.0357 & 0.6980 & 0.0625 & 0.0491 & 2.4667 & 1.4667 & 2.2360 \\
10 & 0.7070 & 0.0277 & 0.7777 & 0.0500 & 0.0361 & 1.4080 & 0.4080 & 0.3954 \\
12 & 0.7729 & 0.0208 & 0.8323 & 0.0384 & 0.0256 & 2.4620 & 1.4620 & 2.1895 \\
\hline
\end{tabular}

Table 3. When $\alpha=1$ and $\lambda=0.5$

\begin{tabular}{ccccccccc}
\hline & \multicolumn{2}{c}{$\tilde{\alpha}$} & \multicolumn{2}{c}{} & \multicolumn{3}{c}{} \\
\hline$n$ & $\tilde{\alpha}$ & $M S E(\tilde{\alpha})$ & $\hat{\alpha}$ & $\operatorname{Bias}(\hat{\alpha})$ & $M S E(\hat{\alpha})$ & $\hat{\lambda}$ & $\operatorname{Bias}(\hat{\lambda})$ & $M S E(\hat{\lambda})$ \\
\hline 5 & 0.9667 & 0.2000 & 1.1279 & 0.1666 & 0.3000 & 0.8953 & 0.3953 & 0.1707 \\
8 & 1.2006 & 0.1428 & 1.3507 & 0.1250 & 0.1964 & 0.4682 & 0.0317 & 0.0586 \\
10 & 1.6277 & 0.1111 & 1.7905 & 0.1000 & 0.1444 & 0.4682 & 0.0317 & 0.0520 \\
12 & 1.5924 & 0.0909 & 1.7251 & 0.0833 & 0.1136 & 1.2191 & 0.7191 & 0.5211 \\
\hline
\end{tabular}

Table 4. When $\alpha=1$ and $\lambda=2$

\begin{tabular}{ccccccccc}
\hline & \multicolumn{2}{c}{$\tilde{\alpha}$} & \multicolumn{2}{c}{} & \multicolumn{3}{c}{} \\
\hline$n$ & $\tilde{\alpha}$ & $M S E(\tilde{\alpha})$ & $\hat{\alpha}$ & $\operatorname{Bias}(\hat{\alpha})$ & $M S E(\hat{\alpha})$ & $\hat{\lambda}$ & $\operatorname{Bias}(\hat{\lambda})$ & $M S E(\hat{\lambda})$ \\
\hline 5 & 1.2415 & 0.2500 & 1.4898 & 0.2000 & 0.4000 & 2.7793 & 0.7793 & 1.2073 \\
8 & 1.0903 & 0.1428 & 1.2266 & 0.1250 & 0.1964 & 3.0306 & 1.0306 & 1.3169 \\
10 & 0.9326 & 0.1111 & 1.0259 & 0.1000 & 0.1444 & 2.0310 & 0.0310 & 0.4920 \\
12 & 1.0497 & 0.0909 & 1.1372 & 0.0833 & 0.1136 & 1.4164 & 0.5835 & 2.0961 \\
\hline
\end{tabular}

From the above tables we observe that for all values of $n$ and $\alpha$, the mean square error of UMVUE of $\alpha$ is less than that of MLE of $\alpha$. Also, as sample size $n$ increases, these mean square errors decrease. 
Table 5. Length of CI of $\alpha, \log (\alpha), \lambda$ and $\log (\lambda)$ when $\alpha=0.5, \lambda=0.5$ and significance level $95 \%$ and $90 \%$

\begin{tabular}{ccccccccc}
\hline & \multicolumn{2}{c}{$\alpha$} & \multicolumn{2}{c}{$\log (\alpha)$} & \multicolumn{2}{c}{$\lambda$} & \multicolumn{2}{c}{$\log (\lambda)$} \\
$n$ & $95 \%$ & $90 \%$ & $95 \%$ & $90 \%$ & $95 \%$ & $90 \%$ & $95 \%$ & $90 \%$ \\
\hline 5 & 1.1759 & 0.98869 & 1.3294 & 1.0766 & 2.8511 & 2.3927 & 9.065 & 5.6372 \\
8 & 0.8333 & 0.6994 & 0.8867 & 0.7307 & 1.8322 & 1.5376 & 2.9829 & 2.1864 \\
10 & 0.7186 & 0.6031 & 0.755 & 0.6245 & 0.6431 & 0.5397 & 0.653 & 0.5456 \\
12 & 0.6401 & 0.5497 & 0.6613 & 0.5497 & 1.3737 & 1.1529 & 1.8065 & 1.4022 \\
\hline
\end{tabular}

Table 6. Length of CI of $\alpha, \log (\alpha), \lambda$ and $\log (\lambda)$ when $\alpha=0.5, \lambda=1$ and significance level $95 \%$ and $90 \%$

\begin{tabular}{ccccccccc}
\hline & \multicolumn{2}{c}{$\alpha$} & \multicolumn{2}{c}{$\log (\alpha)$} & \multicolumn{2}{c}{$\lambda$} & \multicolumn{2}{c}{$\log (\lambda)$} \\
$n$ & $95 \%$ & $90 \%$ & $95 \%$ & $90 \%$ & $95 \%$ & $90 \%$ & $95 \%$ & $90 \%$ \\
\hline 5 & 1.1759 & 0.9869 & 1.4076 & 1.1215 & 3.5481 & 2.9776 & 5.1109 & 3.87 \\
8 & 0.8333 & 0.6994 & 0.8837 & 0.729 & 1.1413 & 0.9578 & 1.1515 & 0.9639 \\
10 & 0.7186 & 0.6031 & 0.7444 & 0.6183 & 1.8756 & 1.5741 & 2.0174 & 1.6573 \\
12 & 0.6093 & 0.5113 & 0.623 & 0.5194 & 0.8932 & 0.7496 & 0.8981 & 0.7525 \\
\hline
\end{tabular}

Table 7. Length of CI of $\alpha, \log (\alpha), \lambda$ and $\log (\lambda)$ when $\alpha=1, \lambda=2$ and significance level $95 \%$ and $90 \%$

\begin{tabular}{ccccccccc}
\hline & \multicolumn{2}{c}{$\alpha$} & \multicolumn{2}{c}{$\log (\alpha)$} & \multicolumn{2}{c}{$\lambda$} & \multicolumn{2}{c}{$\log (\lambda)$} \\
$n$ & $95 \%$ & $90 \%$ & $95 \%$ & $90 \%$ & $95 \%$ & $90 \%$ & $95 \%$ & $90 \%$ \\
\hline 5 & 2.3519 & 1.9738 & 2.6038 & 2.1213 & 3.0361 & 2.5479 & 3.1893 & 2.6381 \\
8 & 1.6667 & 1.3988 & 71.7980 & 1.4758 & 1.9783 & 1.6602 & 2.0136 & 1.6810 \\
10 & 1.4373 & 1.2062 & 1.5577 & 1.2769 & 2.7470 & 2.3054 & 2.9612 & 2.4311 \\
12 & 1.2803 & 1.0745 & 1.3490 & 1.1149 & 5.1938 & 4.3588 & 8.6336 & 6.2941 \\
\hline
\end{tabular}

Table 8. Length of CI of $\alpha, \log (\alpha), \lambda$ and $\log (\lambda)$ when $\alpha=1, \lambda=0.5$ and significance level $95 \%$ and $90 \%$

\begin{tabular}{ccccccccc}
\hline & \multicolumn{2}{c}{$\alpha$} & \multicolumn{2}{c}{$\log (\alpha)$} & \multicolumn{2}{c}{$\lambda$} & \multicolumn{2}{c}{$\log (\lambda)$} \\
$n$ & $95 \%$ & $90 \%$ & $95 \%$ & $90 \%$ & $95 \%$ & $90 \%$ & $95 \%$ & $90 \%$ \\
\hline 5 & 2.0452 & 1.7164 & 2.3371 & 1.8868 & 0.4719 & 0.396 & 0.4774 & 0.3992 \\
8 & 1.6667 & 1.3988 & 1.7745 & 1.4621 & 0.9411 & 0.7898 & 1.1077 & 0.8868 \\
10 & 1.4373 & 1.2062 & 1.4762 & 1.2291 & 0.8855 & 0.7431 & 1.0235 & 0.8236 \\
12 & 1.2803 & 1.0745 & 1.3099 & 1.0919 & 0.2469 & 0.2072 & 0.2473 & 0.2075 \\
\hline
\end{tabular}

From Tables 4 to 8 we observe that as sample size $n$ increases, the length of CIs based on MLE and logtransformed MLE decreases. As reported by Meeker and Escober (1998), we too observe that asymptotic CIs based on log-transformed MLE have better coverage probability.

Table 9. Mean square error of MLE and UMVUE of $\rho(t)$ and length of CI of $\rho(t)$ when $\alpha=2$ and $\lambda=0.5$ at significance level $95 \%$ and $90 \%$

\begin{tabular}{cccccccc}
\hline$t$ & $\rho(t)$ & $\tilde{\rho}(t)$ & $\hat{\rho}(t)$ & $\operatorname{Var}(\tilde{\rho}(t))$ & $M S E(\hat{\rho}(t))$ & $95 \%$ & $90 \%$ \\
\hline 1 & 0.7758 & 0.8062 & 0.7764 & 0.0084 & 0.0105 & 0.4142 & 0.3562 \\
2 & 0.5433 & 0.5772 & 0.5355 & 0.4409 & 0.0211 & 0.2079 & 0.1731 \\
3 & 0.4365 & 0.4768 & 0.4378 & 0.6729 & 0.0216 & 0.0952 & 0.0794 \\
4 & 0.3874 & 0.4244 & 0.3886 & 1.6828 & 0.0209 & 0.0498 & 0.0416 \\
5 & 0.3584 & 0.3928 & 0.3596 & 2.8916 & 0.0202 & 0.0289 & 0.0242 \\
\hline
\end{tabular}




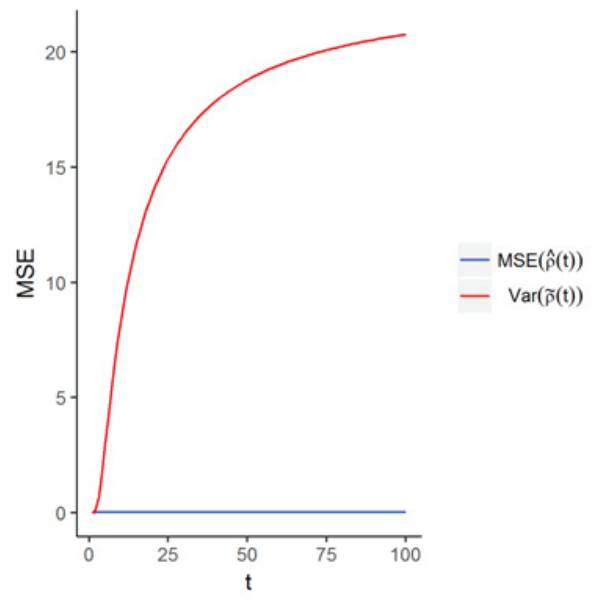

Figure 2. Mean Square Error of MLE and UMVUE of $\rho(t)$.

From Table 9 we observe that as time $t$ increases, the length of CI of $\rho(t)$ based on MLE of $\alpha$ and $\lambda$ decreases. Figure 2 compares the variance of UMVUE of reliability function with the MSE of MLE of reliability function calculated in Table 9 as time $t$ increases. Since the variance of UMVUE of $\rho(t)$ is always greater than the MSE of MLE of $\rho(t)$, thus the MLE of $\rho(t)$ is a more efficient estimator of $\rho(t)$.

In the theory developed in Section 5, for testing the hypothesis $H_{0}: \alpha=\alpha_{0}=2$ against $H_{1}: \alpha \neq \alpha_{0}=2$ under this scheme, we have considered the following sample.

0.14310 .75650 .89031 .59141 .69622 .88554 .72799 .657314 .4171

Now with the help of Chi-Square tables at $5 \%$ level of significance, we obtained $k_{0}=2.0576$ and $k_{0}^{\prime}=7.8815$. Hence, in this case we may accept $H_{0}$ at $5 \%$ level of significance since $U\left(R_{8}\right)=3.7785$.

Again, for testing $H_{0}: \alpha \leq \alpha_{0}=2$ against $H_{1}: \alpha>\alpha_{0}=2$, we have considered the above sample. Now at $5 \%$ level of significance we obtained $k_{0}^{\prime \prime}=2.3476$ and hence, in this case we may accept $H_{0}$ at $5 \%$ level of significance since $U\left(R_{8}\right)=3.7785$.

In order to test $H_{0}: P=P_{0}=0.6666$ against $H_{1}: P \neq P_{0}=0.6666$ under this scheme, we have considered the following Sample $X$ and Sample $Y$.

Sample X: 0.12600 .27550 .36380 .51590 .53161 .03051 .9092

Sample Y: 0.15350 .16530 .24140 .26040 .34260 .44310 .55110 .5709

For these two samples we obtained $U\left(R_{n}\right) / U\left(R_{m}^{*}\right)=2.2445$. Now, with the help of F-tables at $5 \%$ level of significance, we obtained $k_{2}=0.5986$ and $k_{2}^{\prime}=4.9297$. Hence, in this case we may accept $H_{0}$ at $5 \%$ level of significance.

\section{An Example on Real Data}

Lawless (1982) provided real data which represents the number of million revolutions before failure for each of 23 ball bearings in a life test:

$17.88,28.92,33,41.52,42.12,45.6,48.4,51.84,51.96,54.12,55.56,67.8,68.64,68.64,68.88,84.12,93.12$, 98.64, 105.12, 105.84, 127.92, 128.04, 173.4.

Potdar and Shirke (2013) showed that according to Kolmogorov-Smirnov test, this data set best fits generalized inverted half logistic distribution (GIHD). This is also confirmed in the following Figure 3. 


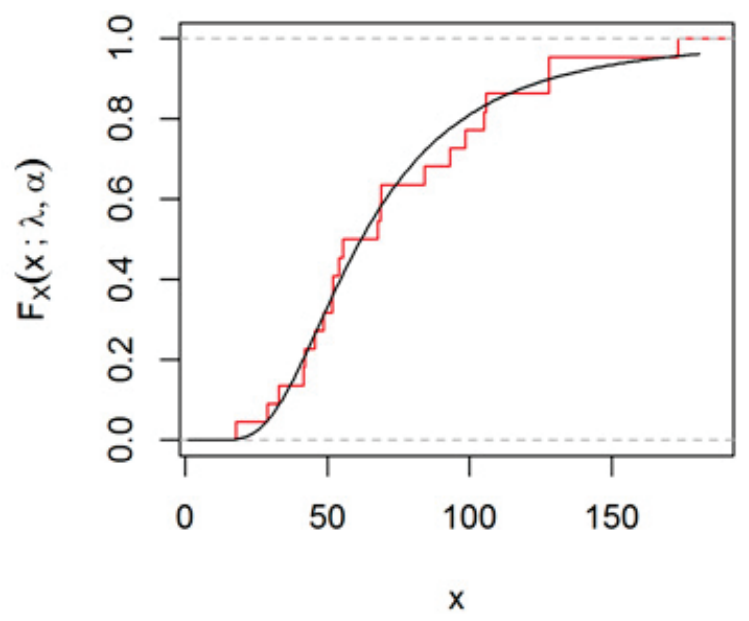

Figure 3. Empirical and Theoretical cdf of GIHD.

In such a case, since both the parameters $\alpha$ and $\lambda$ are unknown, thus by applying Newton Raphson algorithm we obtain the MLES of these parameters. The computed observed Fisher Information matrix is used to obtain the confidence intervals of the parameters and hence the reliability function $\rho(t)$. Table 10 shows the MLE of the parameters and length of CIs based on MLE and log-transformed MLE of $\alpha$ and $\lambda$. We can see that CIs based on $\log$ transformation of MLE of the parameters $\alpha$ and $\lambda$ have a higher coverage probability. In Table 11, MLE and UMVUE of reliability function along with their respective variances and the confidence interval of $\rho(t)$ are computed. We also see that the variance of UMVUE of $\rho(t)$ is smaller than that of its MLE. Hence UMVUE of $\rho(t)$ is more efficient estimator of $\rho(t)$.

Table 10. MLE of $\alpha$ and $\lambda$ and length of CI of $\alpha, \log (\alpha), \lambda$ and $\log (\lambda)$ at significance level $95 \%$ and $90 \%$

\begin{tabular}{cccccccccc}
\hline & \multicolumn{2}{c}{$\alpha$} & \multicolumn{2}{c}{$\log (\alpha)$} & \multicolumn{3}{c}{$\lambda$} & \multicolumn{3}{c}{$\log (\lambda)$} \\
$n$ & $95 \%$ & $90 \%$ & $95 \%$ & $90 \%$ & $95 \%$ & $90 \%$ & $95 \%$ & $90 \%$ \\
\hline 3.2023 & 0.0073 & 4.2370 & 3.5558 & 4.5529 & 3.7413 & 0.00477 & 0.00400 & 0.00485 & 0.00405 \\
\hline
\end{tabular}

Table 11. MLE and UMVUE of $\rho(t)$ and CI of $\rho(t)$ when $t=20$ at significance level $95 \%$ and $90 \%$

\begin{tabular}{cccccc}
\hline$\tilde{\rho}(t)$ & $\operatorname{Var}(\hat{\rho}(t))$ & $\hat{\rho}(t)$ & $\operatorname{Var}(\hat{\rho}(t))$ & $95 \%$ & $90 \%$ \\
\hline 0.9384 & $6.587 \mathrm{E}-07$ & 0.9357 & $7.224 \mathrm{E}-07$ & {$[0.9863,0.9984]$} & {$[0.9874,0.9978]$} \\
\hline
\end{tabular}

\section{Discussion}

This article proposes results on generalized inverted family of distributions having scale and shape parameters. Point and interval estimation procedures for the parameters and reliability characteristics of the family have been developed. As a member of this family, generalized inverted exponential distribution is considered and through simulation techniques, performance of the estimators and confidence intervals are studied. Testing procedures for various parametric functions have been developed. A real life example on generalized inverted half logistic distribution has also been analysed.

Tables 1 to 4 show that for all values of $n$ and $\alpha$, the mean square error of UMVUE of $\alpha$ is less than that of MLE of $\alpha$. Also, as sample size $\mathrm{n}$ increases, these mean square errors decrease. Tables 5 to 8 show that as sample size $n$ increases, we obtain better interval estimates of the parameters of the model under study. As reported by Meeker 
and Escober (1998), we too observe that asymptotic CIs based on log-transformed MLE have better coverage probability. Table 9 shows that as time $t$ increases, we obtain better interval estimates of $R(t)$ based on MLE of $\alpha$ and $\lambda$. Figure 2 compares the mean square error of UMVUE and MLE of reliability function calculated in Table 9 with respect to time $t$. In all we note that the UMVUE of the shape parameter and the reliability function are better estimators than their respective MLES. In all we note that the UMVUE of the shape parameter and the MLE of the reliability function $\rho(t)$ are better estimators than their respective MLE and UMVUE.

\section{Acknowledgement}

We are grateful to the Editor and Reviewers for their valuable comments and suggestions which led to considerable improvement in this paper.

\section{REFERENCES}

1. A.M. Abouammoh, and A.M. Alshingiti, Reliability estimation of Generalized inverted exponential distribution, J. Statist.Comput. Simul, vol. 79, no. 11, pp. 1301 - 1315, 2009.

2. B.C. Arnold, N. Balakrishan, and H. N. Nagaraja, A First Course in Order Statistics, John Wiley \& Sons, New York, 1992.

3. M. Arashi, and M. Emadi, Evidential inference based on record data and inter-record times, Stat.Papers, vol. 49, no. 2, pp. 291 301, 2008.

4. A.M. Awad, and M.K. Gharraf, Estimation of $P(Y<X)$ in the Burr case: A comparative study, Commun. Statist. - Simul., vol. 15 , no. 2, pp. $389-403,1986$.

5. N. Balakrishan, M. Ahsanullah, and P.S. Chan, On the logistic record values and associated inference, Journal of Applied Statistical Science, vol. 2, pp. $233-248,1995$.

6. D.J. Bartholomew, A problem in life testing, Jour. Amer. Statist. Assoc., vol. 52, pp. 350 - 355, 1957.

7. D. J. Bartholomew, The sampling distribution of an estimate arising in life testing, Technometrics, vol. 5, pp. $361-374,1963$.

8. A. P. Basu, Estimates of reliability for some distributions useful in life testing, Technometrics, vol. 6, pp. 215 - $219,1964$.

9. R. A. Belaghi, M. Arashi, and S. M. M. Tabatabaey, On the construction of preliminary test estimator based on record values for the Burr XII model, Communications in Statistics - Theory and Methods, vol. 44, no. 1, pp. 1 - 23, 2015, DOI: $10.1080 / 03610926.2012 .733473$

10. K. N. Chandler, The distribution and frequency of record values, Journal of the Royal Statistical Society, Series B, vol. 14, pp. 220 $-228,1952$.

11. A. Chao, On comparing estimators of $\operatorname{Pr}\{X>Y\}$ in the exponential case, IEEE Trans. Reliability, vol. R-26, pp. $389-392$, 1982.

12. A. Chaturvedi, and T. Kumari, Estimation and testing procedures for the reliability functions of a family of lifetime distributions, interstat.statjournals.net/ YEAR/ 2015/ abstracts/ 1306001.php, 2015.

13. A. Chaturvedi, and A. Malhotra, Estimation and testing procedures for the reliability functions of a family of lifetime distributions based on records, Int. J. Syst. Assur. Eng. Manag., DOI:10.1007/s13198-016-0531-2, 2016.

14. A. Chaturvedi, and A. Malhotra, Inference on the Parameters and Reliability Characteristics of three parameter Burr Distribution based on Records, Appl. Math. Inf. Scien., 11(3), 837-849, 2017.

15. A. Chaturvedi, and A. Pathak, Estimation of the reliability functions for exponentiated Weibull distribution, J. Stat. \& Appl, vol. 7, pp. $1-8,2012$.

16. A. Chaturvedi, and A. Pathak, Bayesian estimation procedures for three parameter exponentiated Weibull distribution under entropy loss function and type II censoring, interstat.statjournals.net/YEAR/2013/abstracts/1306001.php, 2013.

17. A. Chaturvedi, and A. Pathak, Estimation of the reliability function for four-parameter exponentiated generalized lomax distribution, IJSER, Vol. 5, no. 1, pp. 1171 - 1180, 2014.

18. A. Chaturvedi, and U. Rani, Estimation procedures for a family of density functions representing various life-testing models, Metrika, vol. 46, pp. $213-219,1997$.

19. A. Chaturvedi, and U. Rani, Classical and Bayesian reliability estimation of the generalized Maxwell failure distribution, Jour. Statist. Res., vol. 32, pp. $113-120,1998$.

20. A. Chaturvedi, and K. G. Singh, Bayesian estimation procedures for a family of lifetime distributions under squared-error and entropy losses, Metron, vol. 64, no. 2, pp. 179 - 198, 2006.

21. A. Chaturvedi, and K. G. Singh, A family of lifetime distributions and related estimation and testing procedures for the reliability function, Jour. Appl. Statist. Sci., vol. 16, no. 2, pp. 35 - 50, 2008.

22. A. Chaturvedi, and A. Surinder, Further remarks on estimating the reliability function of exponential distribution under type I and type II censorings, Brazilian Jour. Prob. Statist., vol. 13, pp. 29 - 39, 1999.

23. A. Chaturvedi, and S. K. Tomer, Classical and Bayesian reliability estimation of the negative binomial distribution, Jour. Applied Statist Sci., vol. 11, pp. $33-43,2002$.

24. A. Chaturvedi, and S. K. Tomer, UMVU estimation of the reliability function of the generalized life distributions, Statist. Papers, vol. 44, no. 3, pp. $301-313,2003$.

25. Chaturvedi, A., Vyas, S., Estimation and testing procedures for the reliability functions of exponentiated distributions under censorings, Statistica, vol. 77, no. 1, 13-31, 2017. 
26. K. Constantine, M. Karson, and S. K. Tse, Estimation of $P(Y<X)$ in the gamma case, Commun. Statist. - Simul., vol. 15, no. 2, pp. $365-388,1986$.

27. S. Dey, Inverted exponential distribution as a life distribution model from a Bayesian viewpoint, Data Sci. J., vol. 6, pp. 107 - 113, 2007.

28. A. Rdelyi (Ed), Tables of Integral Transforms, McGraw-Hill, 1, 1954.

29. N. Glick, Breaking records and breaking boards, American Mathematical Monthly, vol. 85, pp. 543 - 551, 1978.

30. R. D. Gupta, and D. Kundu, Generalized exponential distribution, Aust. N. Z. J. Statist, vol. 41, no. 2, pp. 173 - $183,1999$.

31. R. D. Gupta, and D. Kundu, Exponentiated exponential family: an alternative to gamma and Weibull distributions, Biom. J., vol. 43, pp. 117 - 130, 2001a. DOI:10.1002/1521-4036(200102)43:1<117::AID-BIMJ117>3.0.CO;2-R

32. R. D. Gupta, and D. Kundu, Generalized exponential distribution: Different methods of estimations, J. Statist. Comput. Simul, vol. 69 , no. 4, pp. $315-338,2001$ b.

33. A. R. Habibi, N. R. Arghami, and J. Ahmadi, Statistical evidence in experiments and in record values, Commun. Stat. Theo. Meth. vol. 35, no. 11, pp. $1971-1983,2006$.

34. N. L. Johnson, Letter to the editor, Technometrics, vol. 17, pp. 393, 1975.

35. G. D. Kelly, J. A. Kelly, ans W. R. Schucany, Efficient estimation of $P(Y<X)$ in the exponential case, Technometrics, vol. 18, pp. $359-360,1976$.

36. H. Krishna, and K. Kumar, Reliability estimation in generalized inverted exponential distribution with progressively type II censored sample, J. Statist. Comput. Simul, vol. 83, no. , pp. 1007 - 1019, 2012.

37. J. F. Lawless, Statistical models and methods for lifetime data, John Wiley and Sons, New York, 1982.

38. E. L. Lehmann, Testing Statistical Hypotheses, John Wiley and Sons, New York, 1959.

39. C. T. Lin, B. S. Duran, and T. O. Lewis, Inverted gamma as life distribution, Microelectron Reliab, vol. 29, no. 4, pp. 619 - 626, 1989.

40. W. Q. Meeker, and L. A. Escober, Statistical Methods for Reliability Data, John Wiley and Sons, New York, 1998.

41. H. N. Nagaraja, Record values and related statistics - A review, Communications in Statistics - Theory and Methods, vol. 17, pp. $2223-2238,1988$ a.

42. H. N. Nagaraja, Some characterizations of continuous distributions based on regressions of adjacent order statistics and record values, Sankhya, Series A, vol. 50, pp. $70-73,1988 b$.

43. K. G. Potdar, and D. T. Shirke, Inference for the scale parameter of lifetime distribution of k-unit parallel system based on progressively censored data, J. Statist. Comput. Simul., DOI: 10.1080/00949655.2012.700314, 2012.

44. K. G. Potdar, and D. T. Shirke, Inference for the parameters of generalized inverted family of distributions, ProbStat, vol. 6 , pp. 18 $-28,2013$.

45. E. L. Pugh, The best estimate of reliability in the exponential case, Operations Research, vol. 11, pp. $57-61,1963$.

46. M. Razmkhah, and J. Ahmadi, Comparing two sampling schemes based on entropy of record statistics, Stat. Papers, vol. 53 , pp. 95 $-106,2011$.

47. V. K. Rohtagi, E. Saleh, and A. K. Md. Ehsanes, An Introduction to Probability and Statistics, Second Edition. John Wiley \& Sons, U.K., 2012.

48. Y. S. Sathe, ans S. P. Shah, On estimating $P(X<Y)$ for the exponential distribution, Commun. Statist. - Theor. Meth., vol. A10, pp. $39-47,1981$.

49. H. Tong, A note on the estimation of $P(Y<X)$ in the exponential case, Technometrics, vol. 16, pp. 625, 1974.

50. H. Tong, Letter to the editor, Technometrics, vol. 17, pp. 393, 1975.

51. R. K. Tyagi, and S. K. Bhattacharya, A note on the MVU estimation of reliability for the Maxwell failure distribution, Estadistica, vol. 41, pp. $73-79,1989$.

52. R. I. Watson, Research design and methodology in evaluating the results of psychotherapy, J. Clin. Psychol., vol. 8, pp. 29 - 33 , 1952. DOI:10.1002/1097-4679(195201)8:1<29::AID-JCLP2270080107>3.0.CO;2-O 